\title{
Weiter- und Fortbildungen
}

\section{Unionstagung der Schweizerischen Gesellschaften für Gefässkrankheiten (USGG), 13.-15. November 2019, in Lu- zern (KKL)}

Die USGG besteht aus den fünf MitgliedGesellschaften:

- Schweizerische Gesellschaft für Angiologie (SGA)

- Schweizerische Gesellschaft für Phlebologie (SGP)

- Swiss Society of Vascular and Interventional Radiology (SSVIR)

- Schweizerische Gesellschaft für Gefässchirurgie (SGG)

- Swiss Society of Microcirculation and Vascular Research (SMVR)

Der Jahreskongress wird jeweils in Kooperation mit der Schweizerischen Gesellschaft für Ultraschall in der Medizin (SGUM), Sektion Gefässe, durchgeführt.

Mitte November richtet die Schweizerische Gesellschaft für Angiologie den 20. USGGJahreskongress in Luzern im Kultur- und Kongresszentrum KKL aus: "20 Jahre USGG - Interdisziplinarität - Heute noch aktuell(er)?». Dr. med. Martin Banyai hat mit einem Team von Co-Chairs ein sehr attraktives Programm mit Schwerpunkt auf das Zusammenspiel der vaskulären Disziplinen zusammengestellt.

PD Dr. D. Heim organisiert das Symposium der Schweizerischen Gesellschaft für Phlebologie unter dem Titel: "Das dicke Bein» (Donnerstag, 14.11.2019, 15:30-17:00 Uhr, im Auditorium).

\section{Venalpina, 26.-31. Jänner 2020, in See-} feld in Tirol

Das Hauptmerkmal der beliebten Weiterund Fortbildungswoche "Venalpina» liegt auf der Praxisrelevanz und der unkomplizierten, freundschaftlichen Atmosphäre unter den Teilnehmern und Referenten. Traditionell organisieren Vorstandsmit- glieder der Österreichischen, Schweizerischen und Deutschen Gesellschaft für Phlebologie den Kongress gemeinsam. Heuer haben Prof. Dr. W. Salmhofer (SGÖ), Prof. Dr. J. Hafner (SGP) und Dr. D. Mühlberger (DGP) die Organisation übernommen. Das Programm ist schon weitgehend fertig gestellt und kann bald aufgeschaltet werden. Es berücksichtigt die festen Bestandteile der phlebologischen Diagnostik und Therapie ebenso wie die Innovationen der letzten drei Jahre, und gibt Einblicke in benachbarte Fachgebiete.

\section{Schriftliche Prüfungen zur Erlangung des Fähigkeitsausweises Phlebologie (USGG) SIWF)}

15.11.2019, KKL Luzern (Anmeldefrist abgelaufen)

06.11.2020 Anlässlich der 21. USGG-Jahrestagung in Interlaken:

Anmeldung bis 30.06.2020 bei Dr. med. jürg Traber, Chefarzt Venenklinik Kreuzlingen

\section{Tarifarisches}

Die Revision des ambulanten Arztarifs Tarmed beansprucht nun bereits mehr als fünf Jahre. Die letzte und finalisierte Version, welche seit Mitte 2019 den Behörden zur Genehmigung vorliegt, trägt den Namen TARDOC. Im Laufe der Tarif-Revision und -Pflege erarbeitete eine Delegation der SGP die phlebologischen Positionen, worüber am Do. 14.11.2019 um 17:00 Uhr Dr. med. P. Kern anlässlich des 20. USGGKongresses berichten wird (KKL, Clubraum 3+4, 17:00-18:00).

Die in der Schweiz häufig angewandte Liste für Mittel und Gegenstände (MiGeL) wurde im Laufe des Jahres 2018 von einer interprofessionellen Expertengruppe überarbeitet und nach einem Genehmigungsprozess 01.04.2019 gültig eingeführt. Die
Aufnahme der Kompressionsstrümpfe der Klasse 1 (18-21 mmHg im B-Mass) wurden von den Behörden nicht genehmigt. Somit werden diese Kompressionsstrümpfe weiterhin nicht rückerstattet. Dagegen wurde folgender Grundsatz in der MiGeL explizit verankert:

"Vergütung nur bei Abgabe durch eine Abgabestelle, die einen Vertrag mit dem Versicherer gemäss Artikel 55 KVV hat, der die notwendigen Qualitätsanforderungen beinhaltet (insbesondere Vermessung der Beine, Anprobe und persönliche Beratung bezüglich Handhabung durch qualifiziertes Personal, regelmässige Kontrolle der Masse). Medizinische Kompressionsstrümp$\mathrm{fe}$, die aufgrund einer durch die versicherten Personen selbst erfolgten Vermessung abgegeben werden, sind nicht leistungspflichtig.»

Diese Vorgaben waren vorbestehend, jedoch den wenigsten explizit bekannt. Nun werden sie besser sichtbar gemacht, und die Ärzteschaft ist aufgefordert, sich damit auseinanderzusetzen. Der SGP-Vorstand stellt sich auf den Standpunkt, dass Phlebologen mit einem gültigen Fähigkeitsausweis für Phlebologie (USGG-SIWF) oder einem Facharztdiplom in Angiologie (SIWF) aufgrund ihrer spezifischen Weiterbildung die notwendige berufliche Qualifikation zur Vermessung und Abgabe medizinischer Kompressionsstrümpfen besitzen. Für die Therapie-Adhärenz der Patienten ist es von Vorteil, wenn die Anpassung, Instruktion und Überprüfung der Kompressionsmittel aus einer Hand erfolgen, und dazu sind Phlebologen genauso gut ausgebildet wie der Fachhandel. Letztlich soll es den Patienten überlassen bleiben, wo sie ihre Kompressionsmittel beziehen wollen. Der Vorstand der SGP wird diese Fragen in den nächsten Monaten weiter klären und wieder berichten. 


\section{Ehrungen}

Anlässlich der 20. Jahrestagung des European Venous Forum (EVF), welches vom 26.-29.06.2019 in Zürich stattfand, wurde Herr PD Dr. med. Dominik Heim hochverdient zum EVF-Präsidenten gewählt. Der
Kongress war ein überaus grosser Erfolg, und von bleibender Erinnerung.

Prof. Dr. med. Jürg Hafner

Präsident Schweizerische Gesellschaft für Phlebologie
IMPRESSUM

Verantwortlich für Mitteilungen der SGP:

Prof. Dr. med. Jürg Hafner, Zürich 